\title{
Dynamic Path Optimization in Sharing Mode to Relieve Urban Traffic Congestion
}

\author{
Hongmei Chen $\mathbb{D}^{1,2}$ and Yuanhang Zhang $\mathbb{D}^{1}$ \\ ${ }^{1}$ School of Economics and Management, Yanshan University, Qinhuangdao 066004, China \\ ${ }^{2}$ Regional Economic Development Research Center, Yanshan University, Qinhuangdao 066004, China
}

Correspondence should be addressed to Hongmei Chen; chenhongmei9@163.com

Received 27 September 2020; Revised 28 April 2021; Accepted 11 May 2021; Published 24 May 2021

Academic Editor: Rodica Luca

Copyright (c) 2021 Hongmei Chen and Yuanhang Zhang. This is an open access article distributed under the Creative Commons Attribution License, which permits unrestricted use, distribution, and reproduction in any medium, provided the original work is properly cited.

\begin{abstract}
In recent years, a new mode of transportation, referred to as "shared commuter buses," has been introduced, which can considerably reduce the dependence of office workers on private cars and relieve urban traffic congestion, but is not widely used owing to efficiency issues. Improving the efficiency of shared commuter buses was set as a goal, and in this study, several research objects were selected to optimize the driving routes of shared commuter buses. The problem of stop location is combined with dynamic route optimization; the "shortest walking distance" model is established to select the most appropriate location for stops. The vehicle routing problem model and "key point updating strategy" are employed to plan routes with real-time traffic information. Finally, we conduct an empirical study to validate our conclusions. The results show that both the model and algorithm are reliable and effective; thus, travel efficiency can be effectively improved and traffic jams can be alleviated.
\end{abstract}

\section{Introduction}

In recent years, the "sharing economy" approach to traffic has become very popular; it has been proved effective in alleviating urban traffic congestion by reducing the usage of private cars; typical examples of such schemes include "shared bicycles" and "shared cars." "Shared commuter bus" schemes can effectively fulfill the commuting needs of urban office workers; however, these schemes are not widely used, predominantly because they cannot ensure efficient travel. Hence, in this study, we attempt to improve the efficiency of shared commuter bus systems by using path optimization. We use the vehicle routing problem (VRP) for reference in our operations research, to optimize the initial routes of commuter buses. However, shared commuter bus systems partially correspond to a mixed-load mode of travel; thus, the traditional VRP model needs to be adjusted.

Psaraftis [1] highlighted several dynamic elements in dynamic VRPs (DVRPs), including "dynamic requirements," "dynamic running times," and "dynamic vehicles." Azi et al. [2] suggested that the dynamic factors of DVRPs can be categorized into two groups based on Psaraftis' theory: the change in customer demand and real-time traffic information. With the development of the Internet of Things and network information technology, information is becoming more readily available; thus, DVRPs based on realtime traffic information have attracted increasing attention from researchers, and these problems are often solved in two stages: initial-route design and dynamic route adjustment. "Initial-route design" is a process of static optimization, and we can solve it in a conventional way; "dynamic route adjustment" is a process of dynamic optimization, in which the most important step is to determine the route-updating strategies.

"Initial-route design" is discussed firstly. Compared with the traditional commuter bus, "shared commuter bus" uses a mixed-load model, in which office workers from different companies can take a ride on the same commuter bus. In the common vehicle routing problem, except for the starting point and the ending point, all nodes in the path network are of the same type. However, in the mixed-load mode of commuter buses, the types of nodes in VRP are different, and the load increases at some nodes and decreases at others, so 
the biggest difficulty lies in the disposal of these nodes. The current research on the mixed-load mode of VRP focuses on the School Bus Routing Problem (SBRP). There is a special class of SBRP, which studies the cooperation of multiple schools to pick up students, and this kind of problem is similar to the content studied in this paper.

"Dynamic route adjustment" is discussed secondly. In the process of "initial-route design," urban road is assumed to be idealized; that is, the influence of other factors except for "path length" on the travel time is not considered, but in the process of "dynamic route adjustment," the factor "realtime traffic information" is considered, and it has a great influence on the final dynamic optimization result.

By consulting numerous documents, we found that the choice of stops strongly influences the final optimization result; however, the existing literature rarely considers this point. In this study, we consider "stop location" as a prerequisite for DVRPs, and we focus on it in our analysis.

In line with the above description, this study proposes a dynamic vehicle routing optimization for a mixed-load mode of operation. The contributions of this study are as follows:

(1) We solve the static VRP for a mixed load and adjust the path dynamically according to the real-time traffic information, and our objective model reflects the focal issues encountered in real-life applications. We designed shared commuter bus routes that improve the vehicle driving efficiencies, consequently rendering the scheme suitable for widespread application as part of the efforts to reduce pressure on urban traffic systems during peak times.

(2) We consider the impacts of stop-location selection on route optimization and effectively integrate the two tasks. Furthermore, we use a clustering algorithm and particle swarm optimization (PSO) algorithm to solve them. The clustering algorithm enables us to identify the most convenient site based on the nearest walking distance; the PSO enables us to determine the most efficient driving route.

The remainder of this paper is organized as follows: Section 2 reviews the relevant literature. Section 3 describes in detail the location model and the vehicle routing optimization model. Section 4 presents the design of the clustering and particle swarm algorithms used to solve the model. Section 5 presents our study on Qinhuangdao as an empirical test case and a specific optimization result. Finally, Section 6 presents the conclusions of this study and recommendations for future work.

\section{Literature Review}

The VRP was proposed by Dantzig and Ramser, and it is widely used in logistic distribution routing problems; there are also some scholars who use it to solve SBRP. However, school buses and commuter buses have different cost and efficiency requirements. Hence, some differences may exist between the two problems in terms of optimization goals and constraint conditions. The DVRP is an important offshoot of the VRP; it was proposed by Psaraftis in 1988; more recently, Psaraftis [3], Bertsimas and Simchi-Levi [4], and Gendreau and Potvin [5] have conducted deeper research on the content of DVRPs. A DVRP based on real-time traffic information is solved in two stages: initial-route design and dynamic route adjustment, and what we need to do in the two stages is to solve a static VRP and choose a route-adjustment strategy.

The static VRP related to the paper is different from traditional VRP, there are few studies on the route optimization of "shared commuter bus," and the mixed-load school bus is similar to "shared commuter buses" in their loading mode, so we mainly refer to the literature studies that study mixed-load SBRP. Ellegood et al. [6] used continuous approximation models to determine whether school bus in mixed-load mode is more beneficial and drew a conclusion that mixed-load mode is more suitable for large districts. Miranda et al. [7] proved that the multiloading approach can substantially reduce cost by practical example analyzing. Mokhtari and Ghezavati [8] introduced a novel mixed-load SBRP and used a biobjective mixed integer linear programming formulation to model it. Shang et al. [9] proposed a time-discretized multicommodity network flow model and transformed the SBRP into a quadratic 0-1 programming model with linear flow balance constraints. Ren et al. [10] considered both school bus stop location and mixed-load routing problem and developed a mixed integer programming model for them.

In order to find the most appropriate route adjust strategy, we identified four different strategies by consulting numerous studies. The first one is the regular updating strategy; principally, this transforms dynamic routes into static routes and occasionally adjusts them. Several scholars have used regular updating strategies in their research (Kilby et al. [11]; Abdallah et al. [12]). The second strategy is that of dynamic event-updating; here, the routes are adjusted when drivers encounter unusual events. Armas and Batista [13] used a dynamic updating strategy in their study to produce a DVRP rich in detail. The third strategy is the docking-site updating strategy; in this scheme, routes are adjusted when vehicles reach distribution nodes. Nakamura et al. [14] used a docking-site updating strategy to choose a dynamic, stochastic route. The fourth strategy is the key point updating strategy. It is the latest but most widely used strategy; its core concept is to define "key points" and adjust routes when vehicles reach these points. Different researchers have used different definitions for "key point," and many scholars have employed the key point updating strategy in their studies (Chen et al. [15]; Li et al. [16]). The Chinese scholar Yanfeng $\mathrm{Li}$, who compared the four strategies under different perturbation frequencies and standard deviations, proved the superiority of the key point updating strategy.

Research on optimization algorithms is more extensive; such algorithms are primarily classified as accurate or heuristic. Accurate algorithms are suitable for the problem which involves less data. Shang et al. [9] decomposed the model into a series of linear multicommodity network flow subproblems when dealing with mixed-load SBRP and used dynamic programming algorithms to solve them. Owing to 
the continuous expansion of research data and the requirements placed upon computational efficiency, heuristic algorithms are a current research hotspot; examples of such algorithms include the tabu search, ant colony, and genetic algorithms, as well as PSO. Hou et al. [17] succeeded to use the algorithm originally developed for pickup and delivery problem with time windows to solve mixed-load SBRP. Wohlgemuth et al. [18] used the tabu search algorithm to plan dynamic routes for disaster rescue vehicles. Li and Chung [19] proposed a new method combining extended insertion and tabu search algorithms to solve VRPs. Schyns [20] used an ant colony algorithm to optimize responsive dynamic vehicle routes. Kuo et al. [21] applied a fuzzy ant colony algorithm to solve DVRPs. Barkaoui and Gendreau [22] designed an adaptive hybrid genetic algorithm by improving the genetic operator; this greatly improved the quality of the genetic algorithm's solution. Abdallah et al. [12] used a genetic algorithm to solve VRPs in which the demand and road condition information are constantly changing. Khouadjia et al. [23] measured the behavior of PSO in terms of dynamic adaptation. Okulewicz and Mańdziuk [24] presented a two-phase multiswarm particle optimizer to solve DVRPs, and they proved its superiority over traditional methods. Jia et al. [25] designed a set-based PSO algorithm to operate a dynamic, logistic dispatching system.

By filtering through and summarizing the existing research, we established a VRP model to solve initial-route design and took the key point updating strategy as the route adjust strategy, owing to its precision. Furthermore, each heuristic algorithm was found to have its own advantages and disadvantages. Thus, we chose the PSO to improve the solving efficiency of models. The stop locations were also considered and $k$-means clustering algorithm was used to find the most suitable stops.

\section{Problem Description and Modeling}

3.1. Problem Description. As important nodes in the path network, the stops have a certain influence on the result of vehicle routing problem. When studying the problem of "stop location," we minimize the walking distance after ensuring all the office workers in need are located within the service range. When dealing with shared commuter bus routing problem, the shared commuter bus's mixed-load attribute is taken into consideration, the office workers on a commuter bus may not have the same destination, and in other words, some middle nodes in the path network are boarding points, which present stop location; others are drop-off points, which present enterprise location, and how to distinguish and process different nodes in VRP mode is the difficulty.

In this study, shared commuter bus routes were optimized to improve driving efficiency. First, we choose appropriate stop locations based on commuters' residences. Second, we determine the service scope and arrival order of each commuter bus, and the reasonable initial routes are thus formed. Finally, we adjust the commuter bus routes using real-time road condition information. In terms of efficiency and comfort requirements of commuters, overlap between different routes and overloading is prohibited. The number of commuters at the stop is assumed to not vary over a short time period, and in other words, another group of DVRP (VRP with dynamic customer demand) is ignored. The general problem studied in this paper can be divided into three subproblems: stop location, initial-route design, and dynamic route adjustment.

Stop location is primarily determined by the number of stops required and commuters' addresses. In this study, commuters' addresses are divided into several regions using a clustering algorithm, and a model based on the shortest walking distance is established. We use the Euclidean distance as a standard model metric; this may result in some disparities between the real scenario and the model, owing to the complexity and irregularity of the actual traffic network; however, we assume that these differences are negligible.

The objective of the initial-route design is to minimize the travel time in an ideal situation. Take the routes of picking up office workers in the morning peak hour as an example, and the nodes in the route network include the stop nodes and the enterprise nodes. Shared commuter buses reach the stop nodes to take on office workers, and reach enterprise nodes to put them off. The VRP model is established to solve the problem, and the schematic diagram is shown in Figure 1. There are often several routes between two stops, and only the shortest one is considered in the process.

The objective of the dynamic route adjustment is to minimize the travel time in a practical situation. In practice, the travel time of the commuter buses cannot be determined by the route lengths, owing to the randomness of congestion and accidents. So, in the process of dynamic route adjustment, we give up the ideal assumption in the initial-route design and take the dynamic traffic information into consideration.

Consistent with the traditional solutions to solve dynamic vehicle routing problems, we divide the process of dynamic path optimization into two steps: static initial-route design and dynamic route adjustment. In addition, the paper makes the following assumption:

(1) Initial-route design is a static optimization process, and when solving it, the road conditions of each route are assumed to be identical; hence, the travel speed of shared commuter bus is fixed, and it should be noted that this assumption only exists in the initial-route design.

(2) The time of stopping at traffic lights is random and unpredictable, and we do not count the time spent on waiting at traffic lights, so it is ignored in this paper. 
(3) Repeated routes will make office workers waste more time, in fact, the shared commuter bus's driving routes are optimized according to the needs of commuters in a short period of time, and ensure the number of commuters at each stop will not exceed the number of seats. So, we will follow the principle and assume that commuters at the same stop are picked up by one commuter bus.

(4) Dynamic route adjustment is a dynamic optimization process, and assumption (1) is invalid when solving it. The problem studied in this paper belongs to DVRP with real-time traffic information, we focus on dynamic path optimization with the changing road conditions, and the change in the number of commuters is not considered.

(5) When shared commuter bus is in motion, real-time dynamic traffic information is available.

In this study, we, thus, solve the three subproblems and develop a "first, set point; then, set line; finally, adjust line" method. In the stop-location problem, the coordinates of commuters' residences are denoted by $Q_{i}\left(X_{i}, Y_{i}\right)$, and we get the coordinates of stops $P_{i}\left(X_{i}, Y_{i}\right)$ by calculating. In the problem of initial-route design, the Euclidean graph is $G=(P, A)$, where $P$ is the collection of nodes and $A$ is the collection of routes, the stop points in $P$ are donated by $P^{*}$, and the enterprise points are $P l, P=P^{*} \cup P^{\prime}$; the distance from the $i$-th residence to the $j$-th residence is $c_{i j}$. In the problem of dynamic route adjustment, the coordinates of key points are denoted by $n_{i}\left(x_{i}, y_{i}\right)$.

\subsection{Mathematical Model}

3.2.1. Parameters. The parameters used in this article are shown in Table 1.

\subsubsection{Decision Variables}

$$
\begin{aligned}
x_{i j s} & = \begin{cases}1, & \text { the } s-\text { th commuter car goes directly from station } i \text { to station } j, \\
0, & \text { otherwise, }\end{cases} \\
y_{i s} & = \begin{cases}1, & \text { the passengers at station } i \text { are served by the } s-\text { th commuter car, } \\
0, & \text { otherwise. }\end{cases}
\end{aligned}
$$

To establish the models from the perspective of the three subproblems, we must establish two models for the first two subproblems and examine the relationship between these two models. The steps used to develop the models are presented in Figure 2.

\subsubsection{Shortest Walking Distance Model for Stop Location.}

By considering office workers' demands for high efficiency during rush hours, we base the stop locations on the principle of maximum convenience, assessing convenience by comparing the walking distances of commuters. Before establishing the shortest walking distance model, the scope of services for each stop must be determined; only then we can know how many clusters commuters' residences are to be sorted into. A $k$-means clustering method is employed in this study; the coordinates of the residences $Q_{i}\left(X_{i}, Y_{i}\right)$ are regarded as the element points to be clustered, and the Euclidean distance is used as the similarity. The clustering process is presented in Figure 3.
Normalization is used to mitigate the influence of the different ranges of elements' abscissa and ordinate values on the clustering. The normalized coordinates of the commuters' residences are labeled as $Q_{i}^{*}\left(X_{i}^{*}, Y_{i}^{*}\right)$. The formulas used to normalize the coordinates are as follows:

$$
\begin{aligned}
X_{i}^{*} & =\frac{X_{i}-\min X_{i}}{\max X_{i}-\min X_{i}}, \\
Y_{i}^{*} & =\frac{Y_{i}-\min Y_{i}}{\max Y_{i}-\min Y_{i}} .
\end{aligned}
$$

After clustering, we assumed that there were five elements (i.e., $A, B, C, D$, and $E$ ) in the first cluster, with the coordinates $\left(x_{A}, y_{A}\right),\left(x_{B}, y_{B}\right),\left(x_{C}, y_{C}\right),\left(x_{D}, y_{D}\right)$, and $\left(x_{E}, y_{E}\right)$, respectively. The stop coordinates were assumed to be $(x, y)$, and the shortest total distance was $D$; the shortest walking distance model was established as follows:

$$
\min D=\sqrt{\left(x-x_{A}\right)^{2}+\left(y-y_{A}\right)^{2}}+\sqrt{\left(x-x_{B}\right)^{2}+\left(y-y_{B}\right)^{2}}+\cdots+\sqrt{\left(x-x_{E}\right)^{2}+\left(y-y_{E}\right)^{2}} .
$$

The optimal solution obtained by solving the shortest walking distance model is an idealized result; real-life urban traffic networks are complicated, and parking is not allowed everywhere. The chosen stop locations must be along roadsides where parking is allowed; hence, we must modify the optimal solution according to the actual traffic situation. 


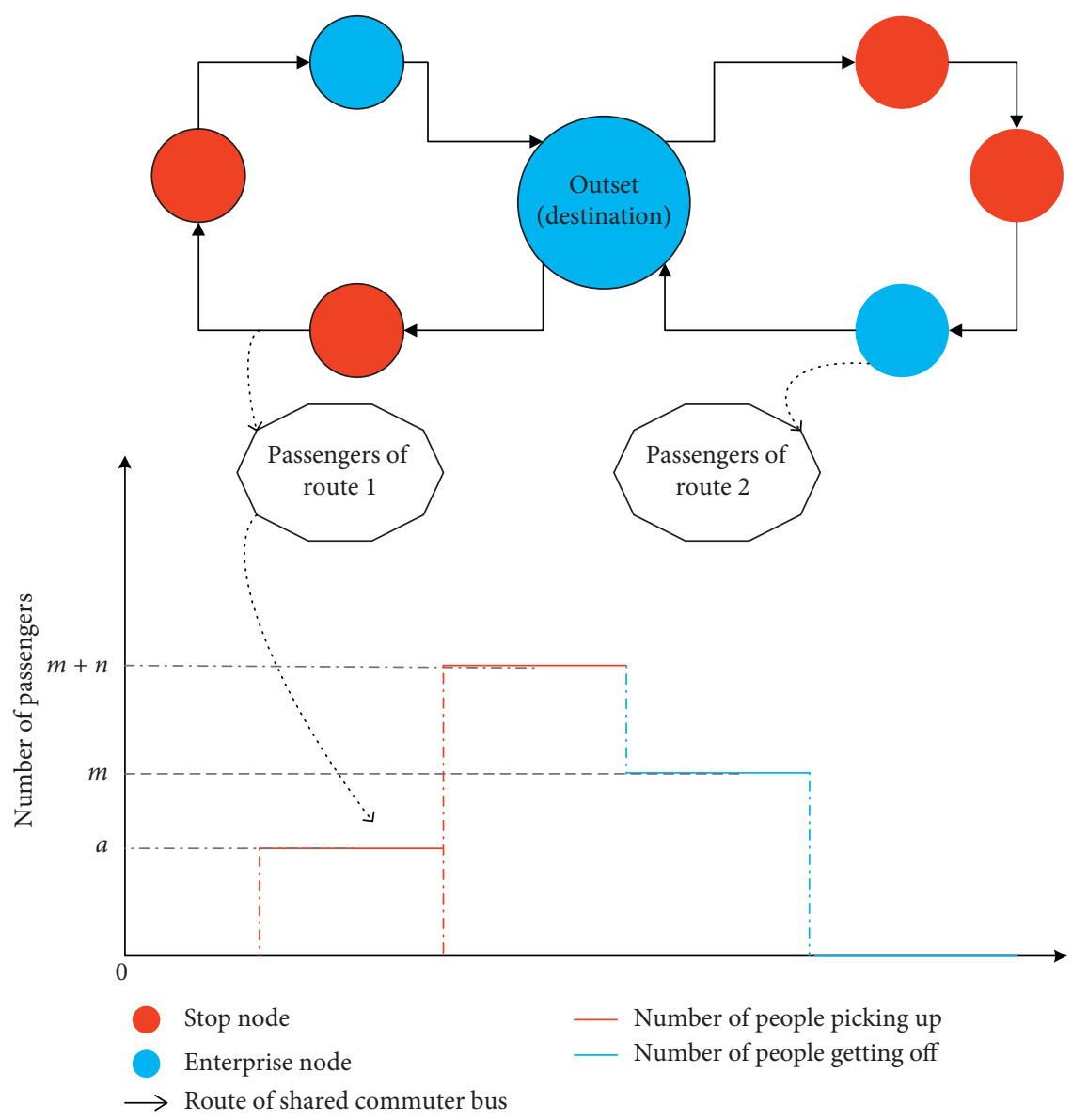

FIgURE 1: Schematic diagram of the VRP in a mixed-load mode.

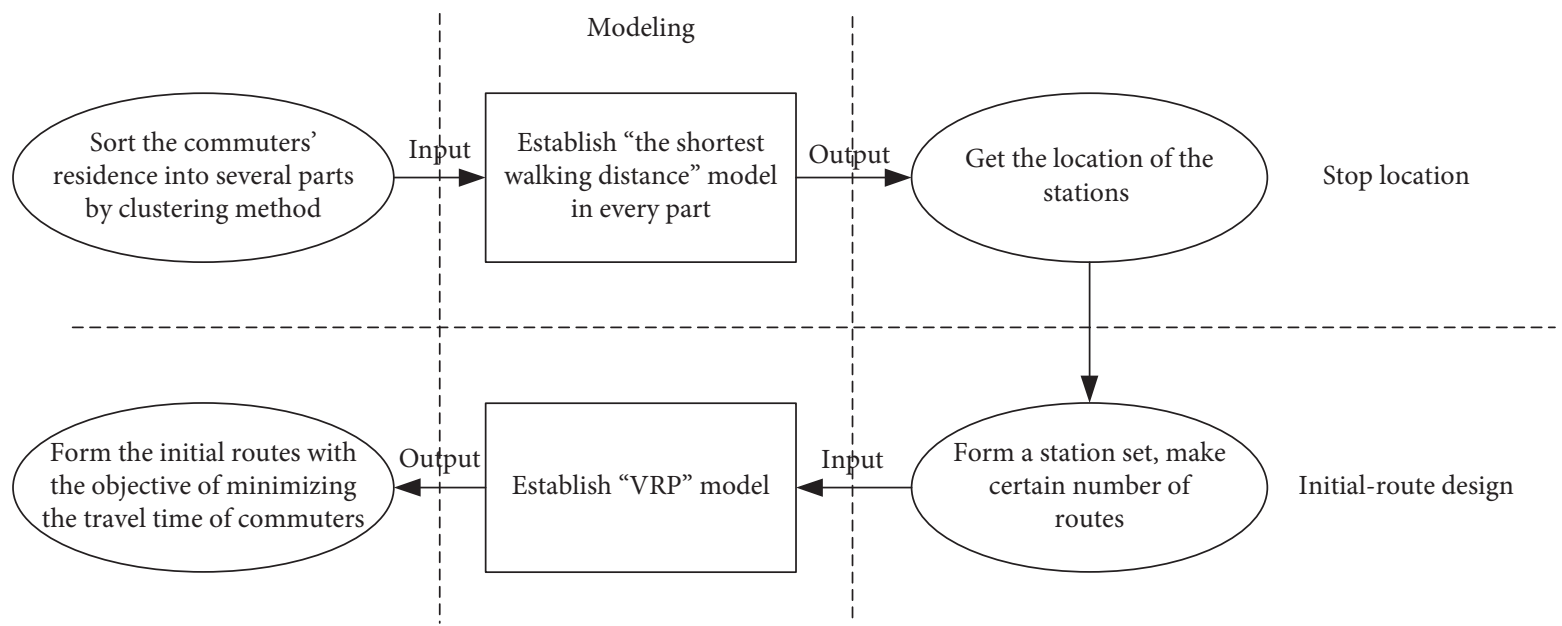

FIGURE 2: Model construction stages.

3.2.4. VRP Model for Initial-Route Design. Considering the importance of delivering high efficiency to commuters during rush hours, the optimization aims to minimize the number of shared commuter buses required and the total driving time. The minimum number of shared commuter buses can be estimated using the following formula:

$$
s=\left\lfloor\frac{\sum P_{i}}{q}\right\rfloor+1 .
$$

To maximize the driving efficiency of shared commuter buses, we introduce a time window, thereby converting the VRP to a VRP with time windows (VRPTW). The total time 
cost is split into the driving time and penalty costs. The Such that influence of other external factors on the total driving time is neglected when designing the initial routes; expressed otherwise, the total distance is the only directly influencing factor of the total driving time. Hence, the objective becomes one of finding the shortest distance. The VRP model is established as follows:

$$
\begin{aligned}
\min Z= & \frac{\sum_{s=0}^{S} \sum_{i=0}^{K} \sum_{j=0}^{K} c_{i j} x_{i j s}}{v} \\
& +e_{1} \sum_{i=1}^{K} \sum_{s=1}^{S}\left\{N \times \max \left[\left(T_{i s}-T_{L i}\right), 0\right]\right\} \\
& +e_{2} \sum_{i=1}^{K} \sum_{s=1}^{S}\left\{M \times \max \left[\left(T_{i s}-T_{L i}\right), 0\right]\right\} .
\end{aligned}
$$

In equation (4), we round down numbers to calculate the minimum number of vehicles required. In equation (5), we take the minimal time cost as our optimization objective and divide the time cost into three parts: travel time; penalty time of soft time windows; penalty time of hard time windows. A penalty factor is multiplied when the soft time limit is exceeded, and this penalty factor becomes an infinite positive number when the hard time limit is exceeded. Equation (6) ensures that the commuter buses are not overloaded, and equation (7) guarantees that each stop is considered. Equations (8) and (9) prevent any duplication of the arrangements. In equation (10), the DFJ method is used to eliminate the subcircuits, $L$ is proper subset of all nodes (stop nodes and enterprise nodes) in the route network, and $|L|$ is the number of elements in $L$. Equation (11) ensures the accuracy of the time window, and equation (12) calculates the time of arrival at the stops and quantifies the penalty costs outside the time window.

\section{Solution Algorithms and Processes}

When solving path optimization problems, the mathematical model can be solved using several methods, including accurate algorithms, commercial optimization packages (e.g., CPLEX), and heuristic algorithms. If the research scale is large, accurate algorithms and commercial optimization packages are too time-consuming; thus, we chose a heuristic algorithm to solve the VRP.

We chose the appropriate algorithms to manage the three subproblems. The change processes of vehicle routes 


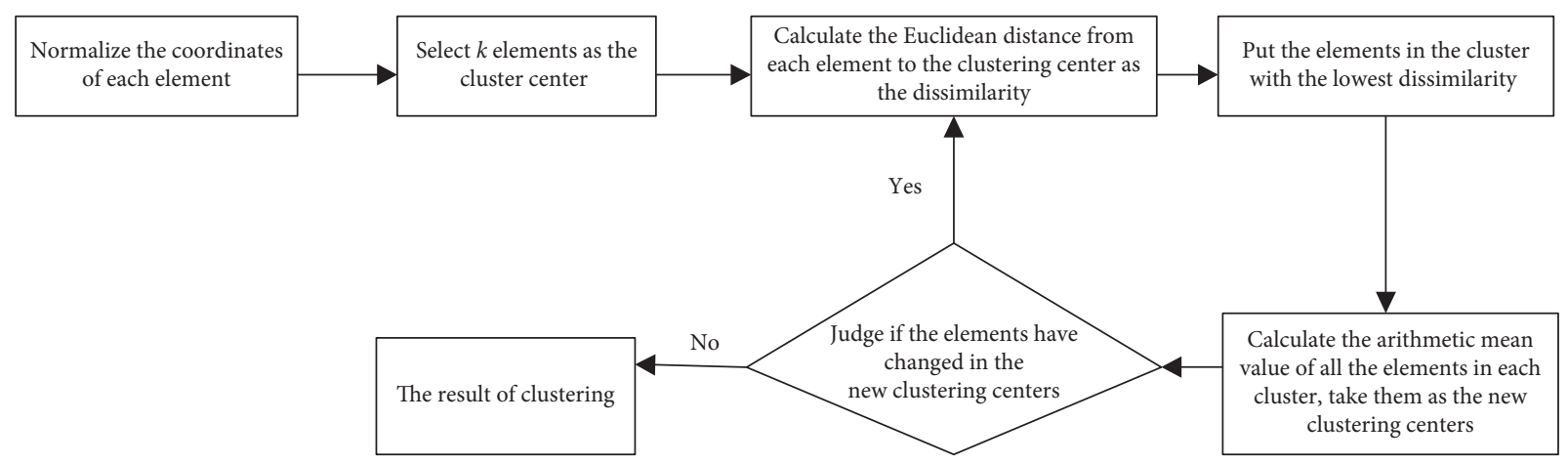

FIGURE 3: Steps of the $k$-means clustering method.

TABle 1: Notations and definitions.

\begin{tabular}{lc}
\hline $\mathbf{Q i}$ & Coordinates of residences \\
$\mathbf{Q i}$ & Normalized coordinates of residences \\
$\mathbf{D}$ & Walking distance from residences to stops \\
$\mathbf{B i}$ & Coordinates of stops \\
$\mathbf{v}$ & The speed of shared commuter bus \\
$\mathbf{p}_{\mathbf{i}}$ & Number of commuters picked up at $i$-th station \\
$\mathbf{d}_{\mathbf{i}}$ & Number of commuters dropped off at $i$-th station \\
$\mathbf{T}_{\mathbf{i s}}$ & Time taken for $s$-th vehicle to reach $i$-th station \\
{$\left[\mathbf{T}_{\mathbf{E i}}, \mathbf{T}_{\mathbf{L i}}\right]$} & Acceptable time window for $i$-th station \\
$\mathbf{N}$ & Unit penalty coefficient beyond soft time window \\
$\mathbf{M}$ & Unit penalty coefficient beyond hard time window \\
$\mathbf{c}_{\mathbf{i j}}$ & The shortest distance from $i$-th node to $j$-th node \\
$\mathbf{n i}$ & Coordinates of key points \\
$\mathbf{X s}, \mathbf{X r}$ & Particle radius vectors \\
$\mathbf{V s}, \mathbf{V r}$ & Particle velocity vectors \\
$\mathbf{p B e s t}$ & Individual extremum \\
$\mathbf{g B e s t}$ & Global extremum \\
$\mathbf{w}$ & Inertial weight \\
$\mathbf{C}$ 1, C2 & Learning effects \\
$\mathbf{r a n d}()$ & Random number between 0 and 1 \\
\hline
\end{tabular}

are shown in Figure 4. For the stop location, we established the shortest walking distance model; then, we chose the method of steepest descent to solve it. For the initial-route design, we established the VRP model, and PSO was used to solve it. For the dynamic route adjustment, we simply used Dijkstra's algorithm in combination with actual road condition information. The specific steps for using the algorithms are presented in Figure 5.

4.1. Stop Location (Steepest Descent Method). The shortest walking distance model was proposed by Cauchy; it is an unconstrained optimization problem containing two variables, and it is often solved by partial derivation. However, when the amount of data is too large to be solved by calculation, the partial derivation is no longer appropriate. The steepest descent method (also referred to as the gradient algorithm) can solve the unconstrained optimization problems with large amount of data quickly, and it is very important to decide the gradient. Since the stop-location objective is to minimize walking distance, we choose a negative gradient, and the feasible solution is iterated (along the negative gradient orientation) using a certain step length, until the lowest point is reached. The initial solution $V_{0}$ and accuracy eps must be determined before using the steepest descent method. In this study, the steepest descent method was implemented in MATLAB to improve the efficiency.

4.2. Initial-Route Design (PSO). PSO is used to find the initial routes, and its steps are as follows.

The first step is to construct the particle expressions. Two questions must be solved for each stop: the first determines which shared commuter bus is responsible for picking up and dropping off the commuters at the specified stop, and the second determines the ordering of the stops that the shared commuter bus visits. The routes can be determined from the number of buses and the order of the stops. The number of buses is represented as $X_{s}$, the stop ordering is represented as $X_{r}$, and the velocity vectors of the particles are represented as $V_{s}$ and $V_{r}$.

The second step is to determine the initial positions and velocities of the particles. In this study, a stop is regarded as a dimension. For the radius vector of each particle, the dimensions of $X_{s}$ take an integer ranging from 1 to $S$, and each dimension of $X_{r}$ takes a real number ranging from 1 to $K$. For the velocity vector of each particle, the dimensions of $V_{s}$ take an integer ranging from $-(S-1)$ to $(S-1)$, and each dimension of $V_{r}$ takes a real number ranging from $-(K-1)$ to $(K-1)$.

The third step is to update the positions and velocities of the particles. The vectors of the particles should be updated continually by comparing the individual extremum pBest with the global extremum gBest; the formulas are given below. The velocity of a particle is represented as $V$, its inertial weight is denoted as $w$, and typically ranges from 0.1 to 0.9 ; learning effects are represented as $C_{1}$ and $C_{2}$, and rand () represents a random number between 0 and 1 : 


$$
\begin{aligned}
V & =w * V+C_{1} * \text { rand } *(p \mathrm{BEST}-\text { Present })+C_{2} * \text { rand } *(g \mathrm{BEST}-\text { Present }), \\
\text { Present } & =\text { Present }+V .
\end{aligned}
$$

4.3. Route Improvement (Dijkstra's Algorithm). By considering the dynamic changes in traffic, an appropriate strategy is developed to adjust the initial routes. The premise of path adjustment is that traffic information can be real-timely obtained, while the rapid development of intelligent transportation systems has made it easy. After comparing applicability of the four kinds of updating strategies, we use the key point updating strategy to adjust the initial routes, and the key points are creatively defined as "road intersections and the stops that the shared commuter buses have not reached in the process of driving" in this paper. The reason we set road intersections as key points is that they play an important role in the urban transportation network. U-turns are not allowed on many roads except at intersections, and the shared commuter buses can promptly adjust their route when they get the real-time traffic information. If we choose other updating strategies, such as the regular updating strategy, then we may not respond quickly to sudden traffic problems; if we choose other traffic points as the key points, there are also some invalid routes to get through after we make the decision to adjust initial routes, which waste the travel time of shared commuter buses.

As shown in Figure 6, suppose the stops are represented as " $B$ " and the key points as " $n$ "; when the shared commuter bus is traversing from $B 1$ to $B 2$, a message describing a sudden accident between $n 1$ and $n 3$ is received; the initial route $B 1-n 1-n 3-B 2$ is adjusted to $B 1-n 1-n 2-n 3-B 2$ using Dijkstra's algorithm. Thus, the route is altered, but the order in which commuters are picked up at each stop remains the same.

Dijkstra's algorithm is used to obtain the shortest route between two points; it is an expedient method for obtaining the shortest routes in unweighted networks; typically, a specific application of Dijkstra's algorithm is achieved using the tentative label $(T)$ and permanent label $(P)$. This study implements Dijkstra's algorithm in MATLAB.

4.4. Empirical Analysis. In China, robust urban rail transit systems are installed in large cities; trams, subways, and other transportation modes release a large portion of highway traffic pressure. Medium and small cities are limited by their original urban layouts; thus, it makes more practical sense to choose medium and small cities to study route optimization for shared commuter buses.

Qinhuangdao is in the northeast of Hebei Province; it borders the Bohai Sea to the south, Yanshan Mountains to the north, Liaoning Province to the east, and Tianjin to the west. It is located on the border between North China and the Northeast China region, at the center of the Bohai Sea Economy Circle. Favorable geographical conditions and rich ecological resources have brought Qinhuangdao numerous development possibilities. In recent years, Qinhuangdao has undergone rapid economic development, achieving a regional gross domestic product of 163.556 billion yuan in 2018. The annual disposable income per capita reached 24,555 yuan, and the annual disposable income of urban households per capita reached 35,386 yuan. This rapid growth of the economy has driven a continuous increase in urban population and improvements in disposable income. Meanwhile, the demand level for services is rising in line with the improvement of material living standards, and urban traffic is one of the most important aspects of this demand. The number of domestic cars in Qinhuangdao has increased significantly in recent years, with the number of private cars rising to 644,000 in 2018 ; this has undoubtedly put immense pressure on the urban traffic system.

In recent years, traffic jam phenomena during rush hours have become increasingly common in Qinhuangdao, and this has had a considerable impact on the travel habits of people. The layout of Qinhuangdao is quite unique: most residential locations are in central Qinhuangdao, and several large industrial sites are located in a remote development zone. Direct buses between these locations are limited in number and slow. Thus, most office workers choose to commute in private cars, and an excessive number of private cars can result in traffic jams. Therefore, optimizing the routes of shared commuter buses will not only improve their commuting efficiency but also effectively reduce the frequency of private car use; this will reduce traffic congestion and relieve urban traffic pressure.

Before beginning path optimization, we conducted a traffic flow analysis on some important road nodes in Qinhuangdao. Through our investigations, we identified several nodes prone to congestion; these are presented in Table 2. Taking these nodes as research objects, we collected their road traffic data over set periods of time, as presented in Table 3.

By analyzing the traffic jam congestion points and traffic flow, it can be observed that traffic jams often occur around Qinhuangdao People's Park. We chose as our research object several residential districts whose residents must pass these traffic jam congestion points to reach their destination.

The algorithms were programmed in MATLAB R2018a

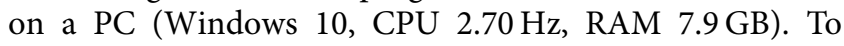
combine them with the actual investigation results, we used the drawing tools to establish a rectangular coordinate system on the map; the points marked on the map were considered the standard of residential information to determine the coordinates of residential locations. The selected residential districts and their corresponding coordinates are presented in Table 4.

Considering the limits of the service range of each stop in Qinhuangdao (500 m-800 m), we determined that four to six stops should be established and took five stops as an example to study firstly; hence, the target number of clusters is five. Before analysis, the coordinates must be normalized; the 


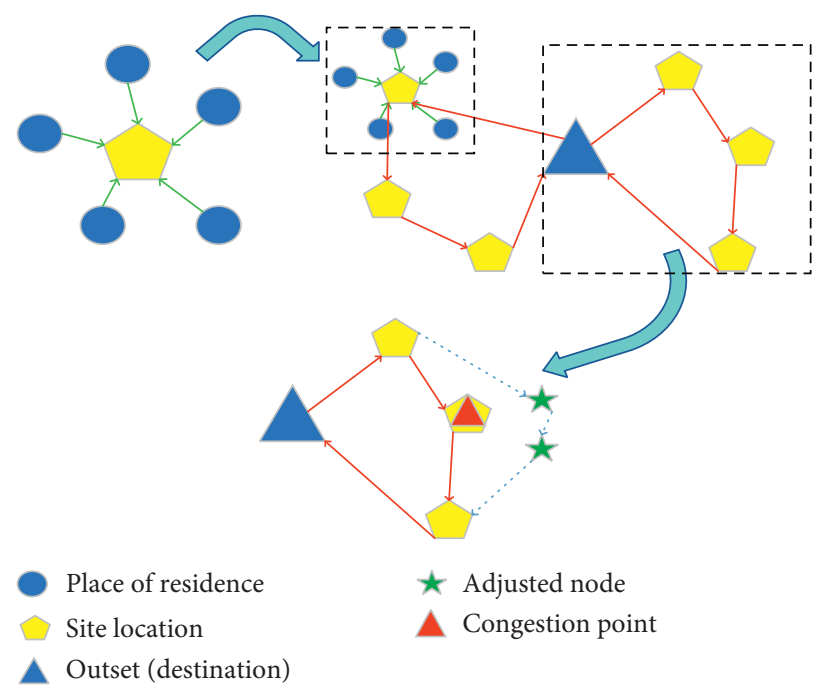

FIgURE 4: Changes in vehicle routes.

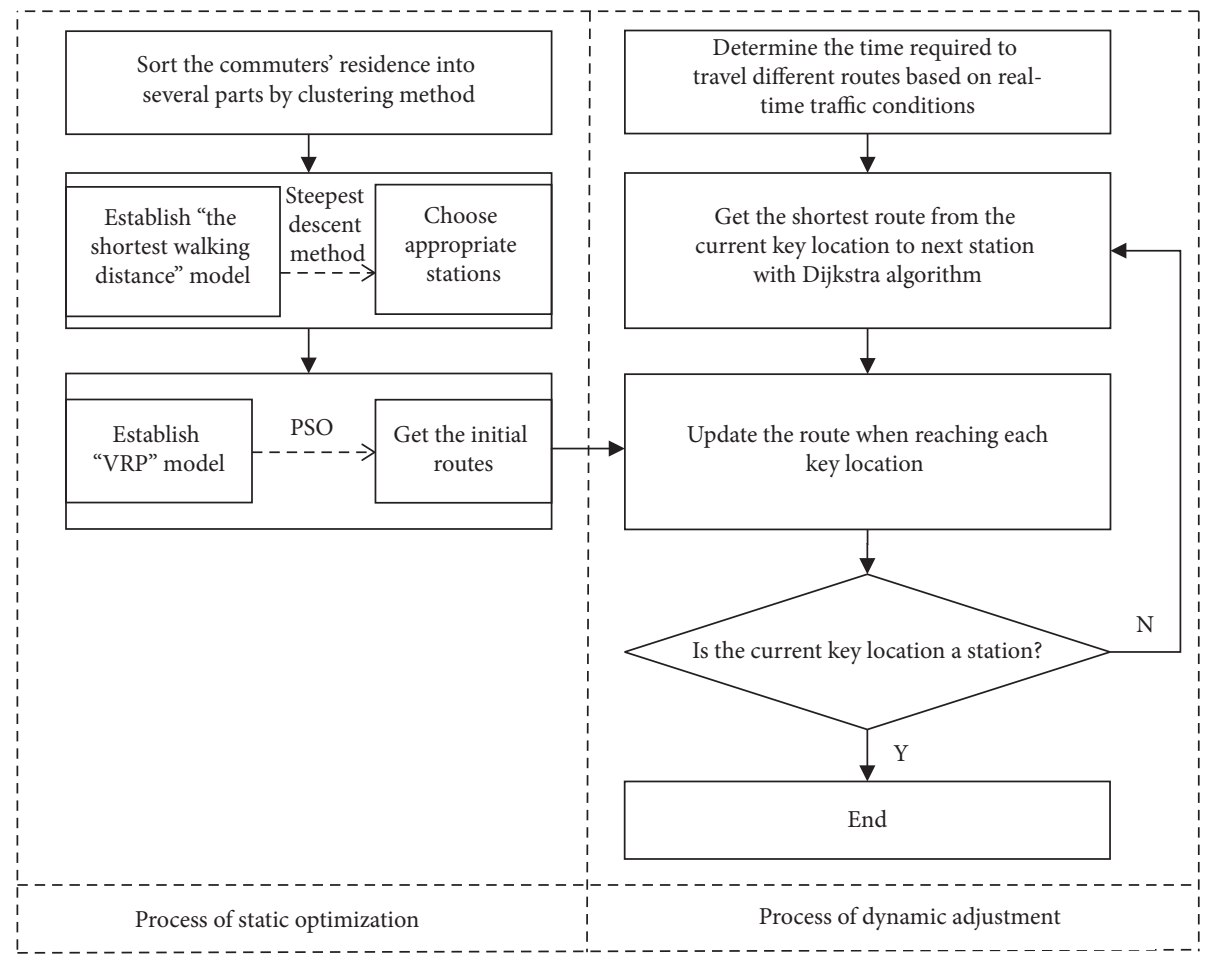

FIgURE 5: Algorithm flowchart.

results are presented (to two decimal places) in Table 4 . Because the choice of the initial clustering center has a large influence on the clustering difficulty, it is necessary to make a preliminary intuitive judgment according to their geographical locations and to select the representative elements. The 1st, 8th, 9th, 12th, and 18th residential districts were selected as the initial clustering centers $a, b, c, d$, and $e$, respectively; five clusters (i.e., $A, B, C, D$, and $E$ ) were created. Using the second residential district (Xuefu) as an example, we calculated the Euclidean distance from this district to the initial clustering centers $a, b, c, d$, and $e$; the results were $0.134164,0.710211,1.113598,0.764199$, and
0.960469 , respectively. Thus, Xuefu is classified as belonging to Cluster $A$. The remaining 14 residential districts were similarly classified. The clustering centers needed to be updated, and their coordinates are as shown in Table 5. We calculated the distances between the 20 residential districts and the five new clustering centers, to cluster again. Finally, the clustering results were not found to change after the third iteration; hence, we considered the final clusters to be stabilized.

For each cluster, a mathematical model based on the shortest walking distance was established. Using Cluster $A$ as an example, the model is expressed as follows: 

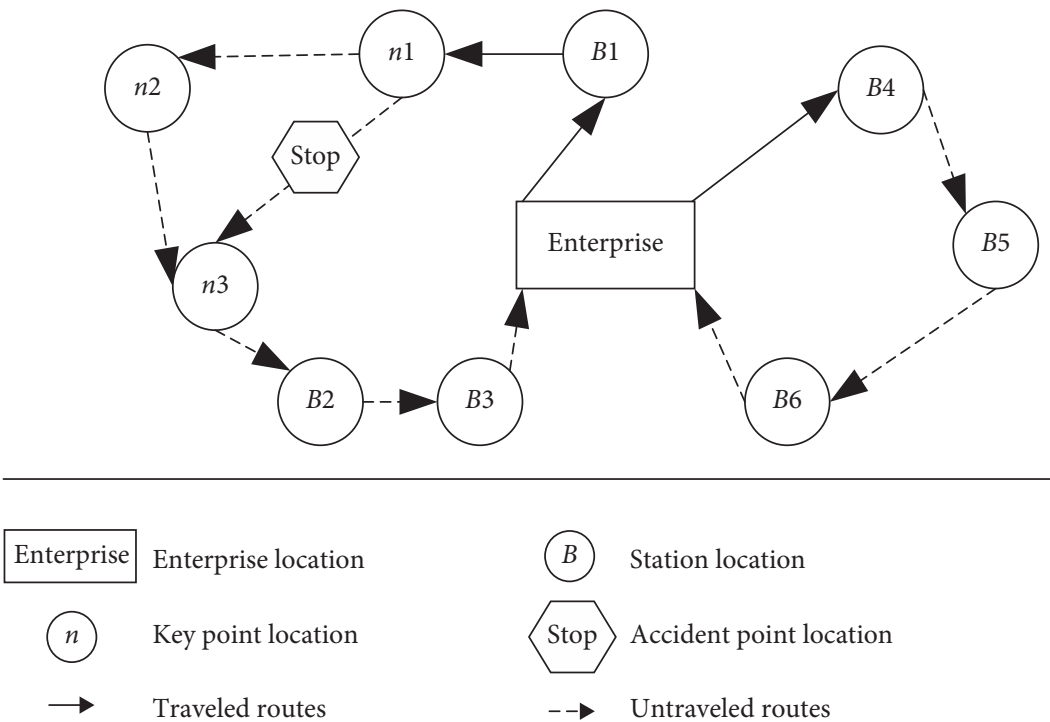

Figure 6: Dynamic adjustment of routes.

TABle 2: Survey of traffic jams in Qinhuangdao.

\begin{tabular}{lccc}
\hline Number & \multicolumn{2}{c}{ Time of traffic jam } & Location of traffic jam \\
\hline 1 & Seven thirty am to eight thirty am & Five pm to six twenty pm & The intersection of Qinhuangxi Street and Haiyang Road \\
2 & Seven thirty am to eight thirty am & Five pm to six pm & The main line of Haiyang Road \\
3 & Seven thirty am to eight thirty am & Four pm to five forty pm & The west section of Qinhuangxi Street \\
4 & Seven thirty am to eight thirty am & Four forty pm to five forty pm & The intersection of Jianshe Street and Wenhua Road \\
5 & Seven thirty am to eight thirty am & Five pm to six twenty pm & The intersection of Hebei Street and Minzu Road \\
6 & Eight am to nine am & Five pm to six twenty pm & The Tanghe Park section of Hebei Street \\
\hline
\end{tabular}

TABle 3: Measured traffic data.

\begin{tabular}{lcccc}
\hline Number & Time & Flow $(\mathrm{veh} / \mathrm{h})$ & Speed $(\mathrm{km} / \mathrm{h})$ & Occupancy $(\mathrm{veh} / \mathrm{km})$ \\
\hline 1 & $8: 00$ a.m. & 73 & 41 & 28 \\
2 & $8: 00$ a.m. & 66 & 48 & 24 \\
3 & $8: 00$ a.m. & 81 & 32 & 32 \\
4 & $8: 00$ a.m. & 72 & 41 & 29 \\
5 & $8: 00$ a.m. & 76 & 39 & 31 \\
6 & $8: 00$ a.m. & 68.00 & 46.00 & 26.00 \\
1 & $5: 30$ p.m. & 53.00 & 52.00 & 13.00 \\
2 & $5: 30$ p.m. & 46.00 & 63.00 & 8.00 \\
3 & $5: 30$ p.m. & 62.00 & 50.00 & 18.00 \\
4 & $5: 30$ p.m. & 57.00 & 58.00 & 10.00 \\
5 & $5: 30$ p.m. & 61.00 & 52.00 & 13.00 \\
6 & $5: 30$ p.m. & 53.00 & 54.00 & 11.00 \\
\hline
\end{tabular}

$$
\begin{aligned}
\min D= & \sqrt{x^{2}+(y-6.6)^{2}}+\sqrt{(x-2.2)^{2}+(y-7.0)^{2}} \\
& +\sqrt{(x-3.5)^{2}+(y-6.2)^{2}}+\sqrt{(x-2.4)^{2}+(y-5.1)^{2}}
\end{aligned}
$$

The model was solved using the steepest descent method in MATLAB. After nine iterations, the coordinates of the chosen stop were $B a(2.2694,6.3406)$. Similarly, the coordinates of the other stops were obtained as $B b(8.3000,2.3000), B c(12.0241$, $0.5150), \quad B d(12.6846,3.6159), \quad$ and $B e(16.5824,2.1458)$.
Finally, the stop locations were adjusted according to the actual road situation; the final choices were $B_{1}$ (Volvo), $B_{2}$ (Bijinghuating), $B_{3}$ (Wantong Building), $B_{4}$ (Student House), and $B_{5}$ (Haisan Building).

The industrial sites in Qinhuangdao are geographically decentralized, and it is too difficult to study all of them; thus, the Qinhuangdao Economic and Technological Development Zone (QETDZ) and Qinhuangdao HR-Channel Management Co., Ltd. (QHRM) were taken as representative of the industrial sites; QETDZ was the beginning and end of all routes, and its coordinates were $E_{1}(9.72,5.86)$; the 
TABLE 4: Coordinates of the 20 selected residential districts.

\begin{tabular}{lccc}
\hline Number & $\begin{array}{c}\text { Residential } \\
\text { district }\end{array}$ & Coordinates & Normalized coordinates \\
\hline 1 & Tiancheng & $(0,6.6)$ & $(0,0.94)$ \\
3 & $3 q u$ & $(3.5,6.2)$ & $(0.19,0.88)$ \\
5 & Gongyuanli & $(6.8,1.8)$ & $(0.37,0.22)$ \\
7 & Bijing & $(8.3,2.3)$ & $(0.45,0.30)$ \\
9 & Jianxinli & $(11.2,0.3)$ & $(0.61,0)$ \\
11 & Shoufu & $(11.5,2.5)$ & $(0.63,0.33)$ \\
13 & Hongweili & $(12.7,2.8)$ & $(0.69,0.37)$ \\
15 & Hejianli & $(13.8,4.7)$ & $(0.75,0.66)$ \\
17 & Hongjingli & $(17.5,0.8)$ & $(0.96,0.07)$ \\
19 & Renminli & $(15.9,3.9)$ & $(0.87,0.54)$ \\
2 & Xuefu & $(2.2,7.0)$ & $(0.12,1.0)$ \\
4 & Guolingwan & $(2.4,5.1)$ & $(0.13,0.72)$ \\
6 & Taisheng & $(7.7,2.0)$ & $(0.42,0.25)$ \\
8 & Hepingli & $(9.2,3.0)$ & $(0.50,0.40)$ \\
10 & Yingqiuxili & $(13.5,0.9)$ & $(0.74,0.09)$ \\
12 & Heanli & $(12.4,3.5)$ & $(0.68,0.48)$ \\
14 & Xianweili & $(10.8,5.0)$ & $(0.59,0.70)$ \\
16 & Fuwubeili & $(15.6,1.6)$ & $(0.85,0.19)$ \\
18 & Kangleli & $(16.0,3.0)$ & $(0.87,0.40)$ \\
20 & Hongqiaoli & $(18.3,3.1)$ & $(1,0.42)$ \\
\hline
\end{tabular}

coordinates of QHRM were $E_{2}(9.63,4.20)$. We investigated the number of office workers required to commute from the chosen residential restricts, and the results are shown in Table 6. In addition, we got the message that the business time of QETDZ and QHRM is half past eight, so we took it as the upper limit of each stop's time window and chose eight as the lower limit. The unit penalty coefficient beyond hard time window $M$ is an infinite number, and the unit penalty coefficient beyond soft time window $N$ is set to 0.25 (15 minutes).

In the VRP model for finding the shortest route, there are two objective constraints: the smallest number of shared commuter buses and the shortest travel time. The priority of the former exceeds that of the latter; thus, the number of required shared commuter buses must be estimated before route optimization. We chose a popular medium-sized coach as the shared commuter bus, which contains 45 seats. The minimum number of shared commuter buses can be calculated as 3 using the previous formula. At the same time, we set $v$ to $20 \mathrm{~km} / \mathrm{h}$ within the allowable speed range for medium-sized coach on urban roads.

Particle swarm optimization was implemented in MATLAB to perform iterative searching. The search dimension $(D)$ should be consistent with the number of shared commuter bus, so it was set to 5 , the learning effects $\left(C_{1}, C_{2}\right)$ were both set to 1.4962 , the inertia weight $(w)$ was set to 0.7298 , the number of particles in the initial swarm $(N)$ was set to 10 , and the maximum number of iterations $(\max D T)$ was set to 100. Finally, we obtained a global extremum ( $g$ Best) of 0.6855 ; the length of the shortest route was found to be $13.71 \mathrm{~km}$. The particle radius vectors are presented in Table 7, from which simple routes can be identified. The first route is from $E_{1}$ to $B_{1}$ to $E_{1}$, the second route is from $E_{1}$ to $B_{2}$ to $E_{2}$ to $E_{1}$, and the third route is from $E_{1}$ to $B_{4}$ to $B_{5}$ to $B_{3}$ to $E_{1}$.
When the number of vehicles increases, the number of particles in the initial swarm should be larger to avoid local optimal solutions. Therefore, we changed the number of particles, setting $N=20,30$, and 40 for the initial swarm. The results obtained were the same as those obtained when $N=10$. In addition, as $w$ increases, the number of iterations required to obtain the optimal path also increases; however, if $w$ is too small, the program will easily fall into a local optimum. We set the inertia weight as $w=0.2298,0.5298$, and 0.8298 and calculated the results. The results were similar to those of the original, and the accuracy of the results could be judged.

To analyze the influence of different stop locations on commuter vehicle path planning, we set the learning factor $c 1=c 2=1.4962$, the inertial weight $w=0.7298$, the number of particles in the initial swarm $N=10$, and the maximum number of iterations $\max D T=100$ (unchanged). We solved for the initial-route design for four and six stops (i.e., the number of clustering targets $k=4$ or 6 and the search dimension $D=4$ or 6 ). The comparison results are shown in Table 8. It can be observed that the initial path length of the design also differs under a change in the number of stops. When four stops were selected, the actual distance was the shortest, and the shared commuter buses' driving efficiency was the highest. When six stops were selected, the actual distance was the longest, and the driving efficiency of the shared commuter buses was the lowest.

In order to verify the optimality of solution found by PSO gives the heuristic nature of this algorithm, we compare the results obtained by using the PSO and the precise algorithm. The exact calculation is implemented in Lingo, and the comparison is shown in Table 9. When the number of stops is set to 4,5 , and 6 , the result of initial-route design found by PSO is equal to the accurate results, but obviously using PSO saves more solution time. In fact, PSO has higher accuracy and faster speed in solving small- and mediumsized optimization problems, but it needs to be improved when large-scale problem comes.

By comparison, the travel time of the shared commuter buses is the shortest when the number of stops is set to 4 , but office workers spend too much time walking to the stops, so we continued our research with five stops. The data for the particle radii vectors can also be restored to the actual expressed node and arrival sequence; the specific initial routes are shown in Figure 7. If more shared commuter buses are required, the number of particles in the initial swarm should be increased to avoid local optimal solutions. No specific regulations exist for the number of initial particles, and we sought to ensure the reliability of the results; thus, we changed the number on the basis of our research; $N$ was set to 20,30 , and 40 to calculate three times. All results were the same as the original ones. Furthermore, with an increase in inertial weight, the iteration time will increase and the computational efficiency will decrease; however, it is easy to fall into a local optimum when the inertia weight is too small, so we adjusted the value of $w$, setting it to 0.2298 , 0.5298 , and 0.8298 . The calculated results are similar to the original ones. This proves the reliability of the empirical results. 
TABLE 5: Results for each clustering step.

\begin{tabular}{lccccc}
\hline Cluster & A (a) & B (b) & C (c) & D (d) & E (e) \\
\hline First cluster & $1,2,3,4$ & $5,6,7,8,11$ & 9,10 & $12,13,14,15$ & $16,17,18,19,20$ \\
Clustering center & $(0,0.94)$ & $(0.50,0.40)$ & $(0.61,0)$ & $(0.68,0.48)$ & $(0.87,0.40)$ \\
Second cluster & $1,2,3,4$ & $5,6,7,8,11$ & 9,10 & $12,13,14,15,19$ & $16,17,18,20$ \\
Clustering center & $(0.11,0.88)$ & $(0.48,0.30)$ & $(0.67,0.04)$ & $(0.68,0.55)$ & $(0.91,0.33)$ \\
Third cluster & $1,2,3,4$ & $5,6,7,8,11$ & 9,10 & $12,13,14,15,19$ & $16,17,18,20$ \\
Clustering center & $(0.11,0.88)$ & $(0.48,0.30)$ & $(0.67,0.04)$ & $(0.72,0.55)$ & $(0.92,0.27)$ \\
\hline
\end{tabular}

TABLE 6: Coordinates of stops and numbers of office workers.

\begin{tabular}{|c|c|c|c|c|c|c|c|}
\hline Stops (enterprise) & $E_{1}$ & $B_{1}$ & $B_{2}$ & $B_{3}$ & $B_{4}$ & $B_{5}$ & $E_{2}$ \\
\hline Coordinates & $(9.72,2.56)$ & $(2.27,6.34)$ & $(8.30,2.30)$ & $(12.02,0.52)$ & $(12.68,3.62)$ & $(16.58,2.15)$ & $(9.62,2.40)$ \\
\hline Number (QETDZ/QHRM) & 0 & $43(43 / 0)$ & $38(13 / 26)$ & $14(14 / 0)$ & $15(15 / 0)$ & $11(11 / 0)$ & 0 \\
\hline
\end{tabular}

TABLE 7: Obtained particle radius vectors.

\begin{tabular}{lcccccc}
\hline Stop & $B_{1}$ & $B_{2}$ & $B_{3}$ & $B_{4}$ & $B_{5}$ & \\
\hline $\mathbf{X}_{\mathbf{s}}$ & 1 & 2 & 3 & 3 & 3 & 2 \\
$\mathbf{X}_{\mathbf{r}}$ & 1 & 1 & 3 & 1 & 2 & 2 \\
\hline
\end{tabular}

TABLE 8: Comparative analysis for different numbers of stops.

\begin{tabular}{|c|c|c|c|c|c|c|}
\hline & & $\begin{array}{l}\text { Number of stops } \\
\text { Global optimal } \\
\text { solution (Gbest) }\end{array}$ & Actual distance $(\mathrm{km})$ & Stop service coverage & Stop-location coordinates & Initial route \\
\hline 4 & $\begin{array}{l}\mathbf{P}_{1} \\
\mathbf{P}_{2} \\
\mathbf{P}_{3} \\
\mathbf{P}_{4}\end{array}$ & $\begin{array}{c}1,2,3,4 \\
5,6,7,8,11 \\
9,10,16,17 \\
12,13,14,15,18,19 \\
20\end{array}$ & $\begin{array}{l}(2.27,6.34) \\
(8.30,2.26) \\
(13.50,0.90) \\
(13.99,3.82)\end{array}$ & $\begin{array}{c}E_{1} \longrightarrow P_{1} \longrightarrow E_{1} \\
E_{1} \longrightarrow P_{2} \longrightarrow E_{2} \longrightarrow E_{1} \\
P_{0} \longrightarrow P_{3} \longrightarrow P_{4} \longrightarrow P_{0}\end{array}$ & 0.6100 & 12.20 \\
\hline 5 & $\begin{array}{l}\mathbf{P}_{1} \\
\mathbf{P}_{2} \\
\mathbf{P}_{3} \\
\mathbf{P}_{4} \\
\mathbf{P}_{5}\end{array}$ & $\begin{array}{c}1,2,3,4 \\
5,6,7,8,11 \\
9,10 \\
12,13,14,15,19 \\
16,17,18,20 \\
\end{array}$ & $\begin{array}{c}(2.27,6.34) \\
(8.30,2.30) \\
(12.02,0.52) \\
(12.68,3.62) \\
(16.58,2.15) \\
\end{array}$ & 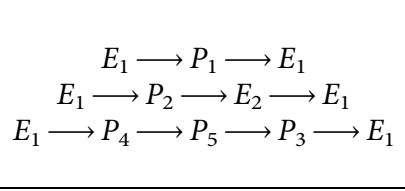 & 0.6855 & 13.71 \\
\hline 6 & $\begin{array}{l}\mathbf{P}_{1} \\
\mathbf{P}_{2} \\
\mathbf{P}_{3} \\
\mathbf{P}_{4} \\
\mathbf{P}_{5} \\
\mathbf{P}_{6}\end{array}$ & $\begin{array}{c}1,2,3,4 \\
5,6,7,8 \\
9,10 \\
11,12,13,15 \\
14 \\
16,17,18,19,20\end{array}$ & $\begin{array}{c}(2.27,6.34) \\
(8.00,2.20) \\
(11.96,0.50) \\
(12.44,3.40) \\
(10.80,5.00) \\
(16.23,2.81)\end{array}$ & $\begin{array}{l}E_{1} \longrightarrow P_{2} \longrightarrow P_{1} \longrightarrow E_{2} \longrightarrow E_{1} \\
E_{1} \longrightarrow P_{1} \longrightarrow P_{5} \longrightarrow E_{2} \longrightarrow E_{1} \longrightarrow P_{3} \longrightarrow E_{1}\end{array}$ & 0.7980 & 15.96 \\
\hline
\end{tabular}

The shared commuter buses will follow the optimized initial routes when picking up and dropping off commuters, accepting the real-time traffic information, and adjusting their route once they reach the key points; it is of crucial importance that we know how long the shared commuter bus will take in each route. In this study, we considered a traffic jam encountered in the investigation process to assess the route-adjustment mechanism; the accident occurred on the Jianshe Road between the Student House and the Haisan Building. As shown in Figure 8, the junctions on the routes were chosen as the key points; the initial route between Student House and Haisan Building is from $B_{4}$ to $n_{1}$ to $n_{2}$ to $n_{3}$ to $n_{4}$ to $B_{5}$; the third shared commuter bus has passed Student House and is approaching the junction of Youyi Road and Jianshe Street. When it reaches $n 1$, the real-time traffic information of the accident is received, and the initial routes are adjusted. The estimated time fed back by the intelligent transportation system for each route is indicated in Figure 8. The route adjustment is solved using Dijkstra's algorithm in MATLAB, and the optimal adjusted route is obtained as running from $P_{4}$ to $n_{1}$ to $n_{6}$ to $n_{3}$ to $n_{4}$ to $P_{5}$; expressed otherwise, the third commuter bus reaches the Haisan Building by passing through Youyi Road, the west section of Heping Street, Hongqi Road, the east section of Jianshe Street, and Wenhua Road, respectively.

\section{Discussion from the Managerial Insight}

Dynamic path optimization is just a part of managing the sharing commuter bus, the main purpose we focus on the shared commuter bus route optimization is to solve the current hot issues, urban traffic jams make commuting 
TABLE 9: Computational results of different algorithms.

\begin{tabular}{lcccc}
\hline & \multicolumn{2}{c}{ PSO } & \multicolumn{2}{c}{ Accurate algorithm } \\
& Result (Gbest) & Running time (second) & Result & 0.6100 \\
Running time (second)
\end{tabular}

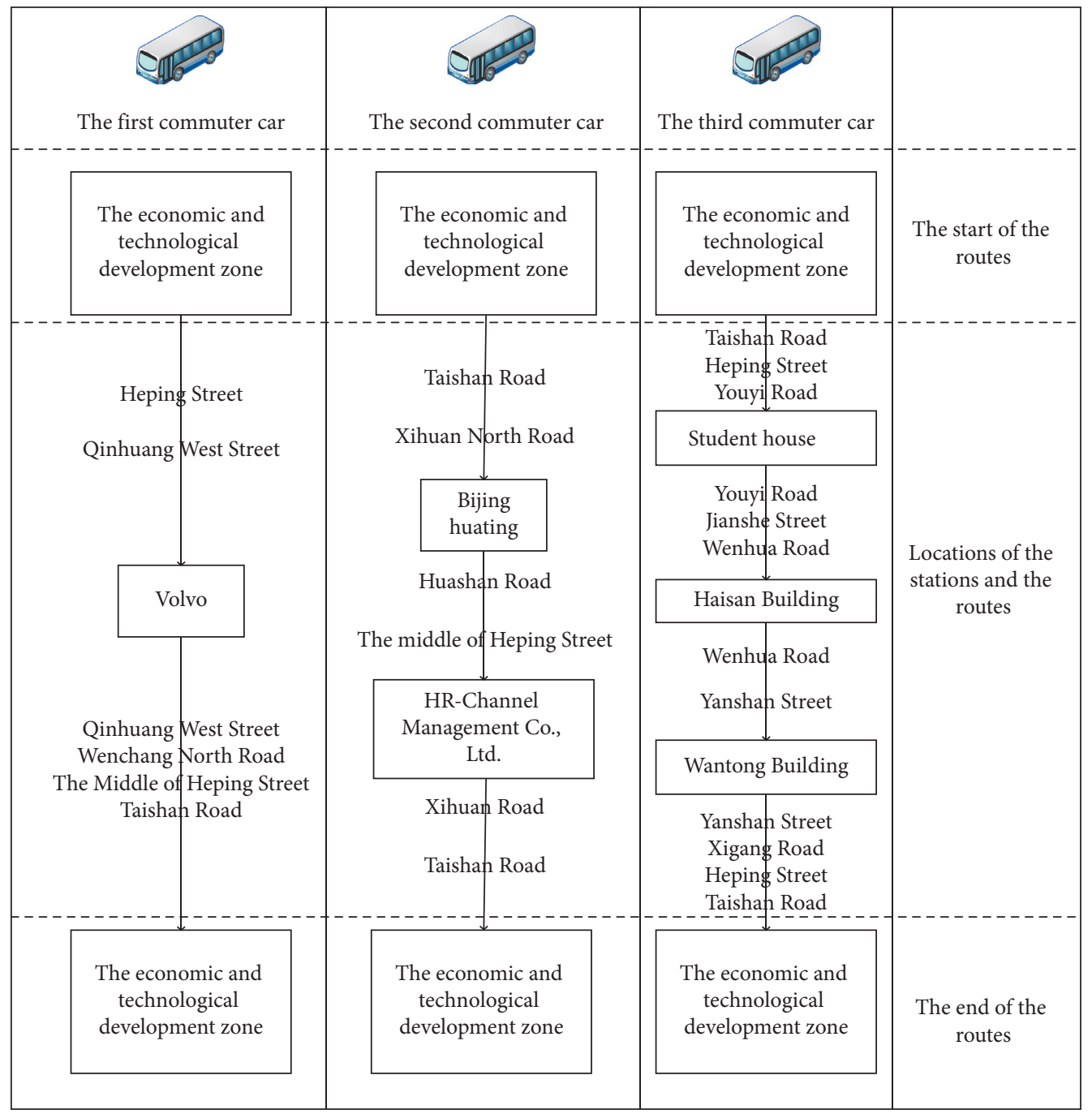

FIgURE 7: Design of initial routes for QETDZ and QHRM.

difficult for commuters, and dynamic route optimization is expected to meet the commuting demand of office workers, to drive office workers choose shared commuter bus, and to reduce their reliance on private cars.

After investigation, we find that there are many idle vehicles in the city at present, and the transport agency can make full use of these resources. The idle vehicles can be rent to provide commuting service of shared commuter bus, which can greatly reduce the waste of idle resources. The managerial insight of dynamic path optimization is shown in Figure 9.

Dynamic path optimization for shared commuter bus belongs to the underlying technology of shared commuter bus, and it provides a more efficient commuting mode for office workers. If an office worker asks for shared commuter bus's services, he may need to schedule on a sharing platform the day before. The platform needs to collect all the reservation data and analyze the data which are submitted to the 


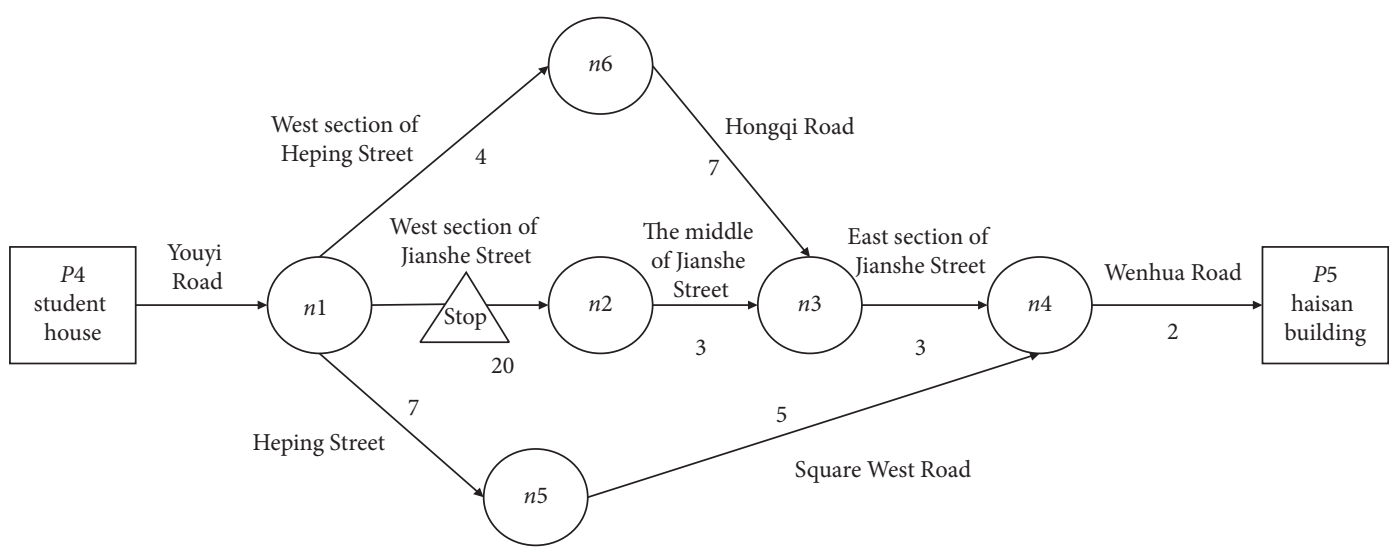

Figure 8: Key points between stops and time consumed on different routes.

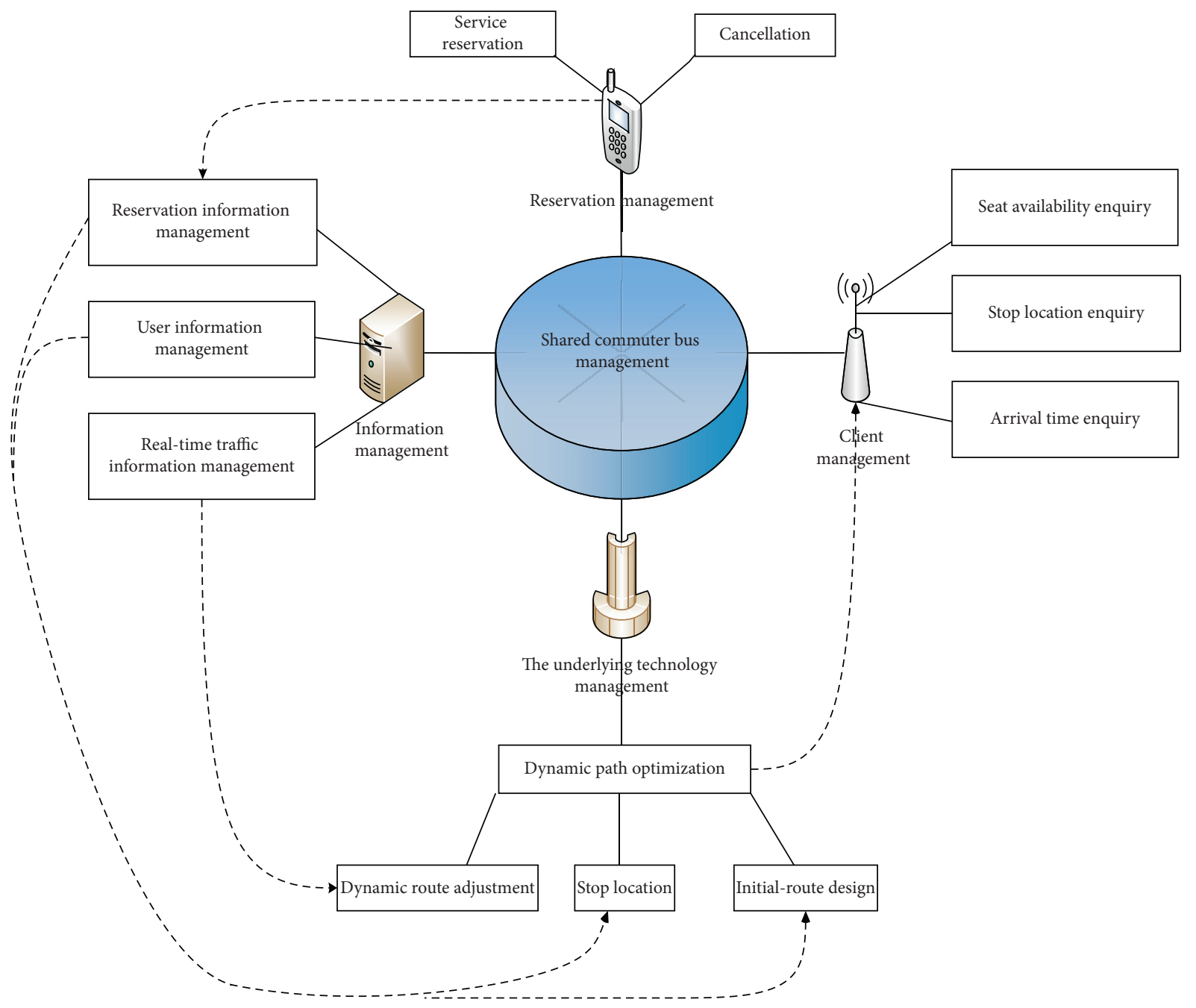

FIGURE 9: The managerial insight of dynamic path optimization.

platform in a same short period of time, then the framework in this paper is used, the stop location is chosen, and the optimal initial routes in an ideal setting are designed; at the same time, the waiting point and time is fed back to the customer. The enough idle vehicles as shared commuter buses also need to be rent to use, the employed drivers are notified to be responsible for the corresponding driving trip. When the shared commuter bus is running, the decision whether it is necessary to adjust the initial route dynamically should be made, and according to the real-time traffic 
information obtained by the intelligent transportation system at each key point, a timely feedback should be given if there is any adjustment in the routes.

In this way, transportation agency can obtain information of commuters timely, use the information to optimize the route, and give the commuters specific feedback. Because of the rapid exchange and processing of information, the shared commuter bus can design the route according to the demand and adjust the route in real time according to the road condition, and transportation mobility in the network can be greatly improved.

\section{Conclusions}

Because the operating routes of shared commuter buses differ from those of other vehicles, this study modified the traditional DVRP model and considered the influence of stop locations on the VRP. To maximize the travel efficiency of shared commuter buses, we studied the DVRP problem using real-time traffic information and established route optimization models that were more appropriate for shared commuter bus. The PSO and Dijkstra's algorithm were used to solve the problem, and their effectiveness was validated through empirical analysis of the QETDZ and QHRM.

In this study, both DVRP and VRPTW were considered when studying the problem of vehicle path optimization. In essence, this is a loadable and unloadable VRP featuring time windows. In the process of solving the initial path, we considered the driving time costs of the vehicle and the penalty costs of exceeding the time window to ensure the highest efficiency; however, this is slightly idealistic because certain other factors are neglected (e.g., site service time costs). Therefore, the mathematical models should be further improved to ensure high accuracy.

\section{Data Availability}

The data used to support this study have been deposited in the Baidu Netdisk; readers can access the data at https://pan. baidu.com/s/1HLq7cur3oHGgvWZZDTBcIQ.

\section{Conflicts of Interest}

The authors declare that there are no conflicts of interest regarding the publication of this paper.

\section{Acknowledgments}

The authors would like to thank Editage (http://www. editage.cn) for the English language editing. The authors would like to acknowledge the support from the Social Science Foundation of Hebei Province, China (Grant no. HB20GL2026).

\section{References}

[1] H. N. Psaraftis, Vehicle Routing: Methods and Studies, Elsevier, Amsterdam, Netherlands, 1988.

[2] N. Azi, M. Gendreau, and J.-Y. Potvin, "A dynamic vehicle routing problem with multiple delivery routes," Annals of Operations Research, vol. 199, no. 1, pp. 103-112, 2012.
[3] H. N. Psaraftis, "Dynamic vehicle routing: status and prospects," Annals of Operations Research, vol. 61, no. 1, pp. 143-164, 1995.

[4] D. J. Bertsimas and D. Simchi-Levi, "A new generation of vehicle routing research: robust algorithms, addressing uncertainty," Operations Research, vol. 44, no. 2, pp. 286-304, 1996.

[5] M. Gendreau and J.-Y. Potvin, "Dynamic vehicle routing and dispatching," Fleet Management and Logistics, Springer, Berlin, Germany, pp. 115-126, 1998.

[6] W. A. Ellegood, J. F. Campbell, and J. North, "Continuous approximation models for mixed load school bus routing," Transportation Research Part B: Methodological, vol. 77, no. 1, pp. 182-198, 2015.

[7] D. M. Miranda, R. S. de Camargo, S. V. Conceição, M. F. Porto, and N. T. R. Nunes, "A multi-loading school bus routing problem," Expert Systems with Applications, vol. 101, no. 1, pp. 228-242, 2018.

[8] N.-a. Mokhtari and V. Ghezavati, "Integration of efficient multi-objective ant-colony and a heuristic method to solve a novel multi-objective mixed load school bus routing model," Applied Soft Computing, vol. 68, no. 1, pp. 92-109, 2018.

[9] P. Shang, L. Yang, Z. Zeng, and L. Tong, "Solving school bus routing problem with mixed-load allowance for multiple schools," Computers \& Industrial Engineering, vol. 151, Article ID 106916, 2021.

[10] J. Ren, W. Jin, and W. Wu, "A two-stage algorithm for school bus stop location and routing problem with walking accessibility and mixed load," IEEE Access, vol. 7, no. 1, pp. 119519-119540, 2019.

[11] P. Kilby, P. Prosser, and P. Shaw, Dynamic VRPs: A Study of Scenarios, University of Strathclyde, Glasgow, UK, 1998.

[12] A. M. F. M. Abdallah, D. L. Essam, and R. A. Sarker, "On solving periodic re-optimization dynamic vehicle routing problems," Applied Soft Computing, vol. 55, pp. 1-12, 2017.

[13] J. D. Armas and B. M. Batista, "Variable neighborhood search for a dynamic rich vehicle routing problem with time windows," Computers \& Industrial Engineering, vol. 85, pp. 120-131, 2015.

[14] Y. Nakamura, E. Taniguchi, and T. Yamada, "Selecting a dynamic and stochastic path method for vehicle routing and scheduling problems," in Proceedings of the 6th International Conference on City Logistics, vol. 2, no. 3, pp. 6042-6052, Puerto Vallarta, Mexico, 2009.

[15] H.-K. Chen, C.-F. Hsueh, and M.-S. Chang, "The real-time time-dependent vehicle routing problem," Transportation Research Part E: Logistics and Transportation Review, vol. 42, no. 5, pp. 383-408, 2006.

[16] Y. F. Li, Z. Y. Gao, and J. Li, "Vehicle routing problem in dynamic urban network with real-time traffic information," Systems Engineering-Theory \& Practice, vol. 33, no. 7, pp. 1813-1819, 2013.

[17] Y.-E. Hou, L. Dang, W. Dong, and Y. Kong, "A metaheuristic algorithm for routing school buses with mixed load," IEEE Access, vol. 8, no. 1, pp. 158293-158305, 2020.

[18] S. Wohlgemuth, R. Oloruntoba, and U. Clausen, "Dynamic vehicle routing with anticipation in disaster relief," SocioEconomic Planning Sciences, vol. 46, no. 4, pp. 261-271, 2012.

[19] Y. Li and S. H. Chung, "Disaster relief routing under uncertainty: a robust optimization approach," IISE Transactions, vol. 51, no. 8, pp. 869-886, 2019.

[20] M. Schyns, "An ant colony system for responsive dynamic vehicle routing," European Journal of Operational Research, vol. 245, no. 3, pp. 704-718, 2015. 
[21] R. J. Kuo, B. S. Wibowo, and F. E. Zulvia, "Application of a fuzzy ant colony system to solve the dynamic vehicle routing problem with uncertain service time," Applied Mathematical Modelling, vol. 40, no. 23-24, pp. 9990-10001, 2016.

[22] M. Barkaoui and M. Gendreau, "An adaptive evolutionary approach for real-time vehicle routing and dispatching," Computers \& Operations Research, vol. 40, no. 7, pp. 17661776, 2013.

[23] M. R. Khouadjia, B. Sarasola, E. Alba, L. Jourdan, and E.-G. Talbi, "A comparative study between dynamic adapted PSO and VNS for the vehicle routing problem with dynamic requests," Applied Soft Computing, vol. 12, no. 4, pp. 1426-1439, 2012.

[24] M. Okulewicz and J. Mańdziuk, "The impact of particular components of the PSO-based algorithm solving the dynamic vehicle routing problem," Applied Soft Computing, vol. 58, pp. 586-604, 2017.

[25] Y.-H. Jia, W.-N. Chen, T. Gu et al., "A dynamic logistic dispatching system with set-based particle swarm optimization," IEEE Transactions on Systems, Man, and Cybernetics: Systems, vol. 48, no. 9, pp. 1607-1621, 2018. 